\title{
Detection of Diurnal and Semidiurnal Tidal Signatures from Continuous GPS Daily Vertical Residual Time Series by Frequency Mixing Method
}

\author{
Yu Peng, ${ }^{1,2,3}$ Wen Chen, ${ }^{1,2,3^{*}}$ Danan Dong, ${ }^{1,2,3}$ Chao Yu, ${ }^{1,2,3}$ \\ Zhiren Wang, ${ }^{1,2,3}$ Jun Yan, ${ }^{1,2,3}$ and Min Liu ${ }^{2,4}$ \\ ${ }^{1}$ Engineering Center of SHMEC for Space Information and GNSS, East China Normal University, \\ Shanghai 200241, China \\ ${ }^{2}$ Key Laboratory of Geographic Information Science, Ministry of Education, East China Normal University, \\ Shanghai 200241, China \\ ${ }^{3}$ Shanghai Key Laboratory of Multidimensional Information Processing, East China Normal University, \\ Shanghai 200241, China \\ ${ }^{4}$ Institute of Eco-Chongming, East China Normal University, Shanghai 200241, China
}

(Received December 30, 2018; accepted March 8, 2019)

Keywords: CGPS, diurnal and semidiurnal tidal signatures, tidal displacements, harmonic analysis, frequency mixing, time series

The diurnal and semidiurnal tidal displacements in the continuous GPS (CGPS) daily coordinate time series cannot be perfectly removed by ocean tide models, especially in coastal regions. The residual tidal displacements will propagate to the daily time series as alias signals with longer periods ranging from about 2 weeks to 1 year, which can be theoretically calculated. However, the alias signals with long periods (e.g., more than half a year) are difficult to detect through alias harmonic analysis when the length of the time series is insufficient. To extract all the diurnal and semidiurnal tidal displacements from the daily coordinate time series, we propose the frequency mixing method, that is, converting the signals from a high frequency to a low frequency, before the harmonic analysis. We demonstrate the feasibility of this method using simulated data and analyze the spectra of real daily vertical residual time series from eight globally distributed CGPS stations. The solar-related ocean tidal constituents (K1, P1, K2, and S2) are clearly detected from the time series by this method, verifying that the FES2004 model on solar-related ocean tidal displacement should be improved.

\section{Introduction}

The joint contribution of the centrifugal force from the Earth's revolution and the gravitational attraction of the moon and sun generates the periodic surface displacement of the solid Earth as well as the periodic migration of oceanic mass, which are called solid Earth tide (or Earth body tide, EBT) and ocean tide loading (OTL), respectively. ${ }^{(1,2)}$ These tides alter the rotational and translational statuses of the solid Earth, and are absorbed by earth rotation

*Corresponding author: e-mail: wchen@sist.ecnu.edu.cn https://doi.org/10.18494/SAM.2019.2252 
parameters (ERPs) and geocenter parameters, ${ }^{(3,4)}$ and also cause site displacements in the solid Earth-fixed frame (CE frame); such displacements appear in the time series of continuous GPS (CGPS) station coordinates. The EBT causes vertical displacements of more than dozens of centimeters in low-latitude regions, while the OTL can generate additional vertical displacements of over $10 \mathrm{~cm}$ in coastal regions. These periodic site displacements caused by the EBT and OTL are conventionally modeled during CGPS data processing. The EBT and three long-period OTL effects (Mf, Mm, and Ssa) are considered to be well modeled to an accuracy of around $1 \%$ according to the IERS Conventions 2010. ${ }^{(5-8)}$ However, owing to the inaccuracy of current tide models, the diurnal and semidiurnal tidal signatures still remain in the daily coordinate time series, especially in the coastal areas. Taking the Weddell Sea and Ross Ice Shelf as an example, the root mean square (RMS) of the vertical amplitude difference of various tide models can reach $8 \mathrm{~mm}$ at some International GNSS Service (IGS) sites. ${ }^{(9)}$

The increasing number of globally distributed CGPS stations and the advances in the method of data analysis make the GPS technique particularly effective in validating ocean tide models. Scientists have started to use CGPS observations to improve the accuracy of ocean tide model predictions. ${ }^{(10-12)}$ Current approaches to estimating the tidal displacements using CGPS solutions fall into three categories: the harmonic estimation approach (static approach), the kinematic approach, and the alias harmonic analysis. ${ }^{(9,12-14)}$

The harmonic estimation approach (hereafter, the static approach) solves the harmonic displacement coefficients of the primary (usually 8) tidal constituents as additional parameters during the data processing of the static GPS batch solution (typically daily). This approach was first developed in VLBI data analysis, ${ }^{(15,16)}$ and then adopted in CGPS data analysis by Schenewerk et al. ${ }^{(17)}$ In early analysis, the misfit between their GPS-estimated M2 frequency height displacement amplitudes and the Schwiderski ocean tide model prediction was within $5 \mathrm{~mm} .{ }^{(17)}$ Then, King and co-workers used such a static approach to estimate the GPS observed tidal displacements and compare them with FES99 ocean tide model predictions. They found that the discrepancies between the GPS-estimated M2 height amplitudes and the model predictions were reduced to sub-mm levels. ${ }^{(18,19)}$ Yuan et al. used a similar approach to investigate the tidal displacements of 456 globally distributed CGPS stations and compared their results with the predictions of eight recent global ocean tide models, namely, CSR4.0, NAO99b, FES2004, TPXO7.2, HAMTIDE11a, DTU10, EOT11a, and GOT4.7. They demonstrated that the accuracy of their GPS displacement estimations is better than $\pm 0.24 \mathrm{~mm}$ for the lunar-only constituents (M2, N2, O1, and Q1), which is suitable for providing constraints on the internal structure of the Earth. ${ }^{(20)}$

The kinematic approach applies the kinematic GPS techniques to obtain subdaily $(\leq 2 \mathrm{~h})$ coordinate time series from which the harmonic coefficients of tidal displacements can be estimated by a conventional harmonic analysis. This approach was first demonstrated by Khan and Tscherning, who obtained the M2 height amplitudes from GPS hourly coordinate time series spanning 49 days. The discrepancies between their estimates and the GOT99.2 ocean tide model predictions are less than $3.4 \mathrm{~mm} .{ }^{(21)}$ Similar subsequent studies have been carried out for different regions. The resulting M2 height amplitudes deviate from the various ocean tide model predictions by about $1-4 \mathrm{~mm} \cdot{ }^{(14,22-24)}$ Using the postprocessed kinematic precise point 
positioning GPS with appropriately tuned process noise constraints, Penna et al. demonstrated that the periodic displacements can be reliably observed at the $0.2 \mathrm{~mm}$ level, which is sufficient to test and refine the ocean tide and solid Earth response models. ${ }^{(11)}$

Although the accuracy of both the static and kinematic approaches have reached the submillimeter level, neither of them can directly use the daily coordinate time series provided by IGS data analysis centers. They require the reprocessing of the observation data with huge workloads as well as a long data collection span (at least 10 years) to reduce the high correlation with the atmospheric zenith delay parameters and potential interference from seasonal mass loading. The vast amount of daily coordinates time series provided by the IGS analysis centers has been used in various geophysical studies, such as those on crustal deformation, plate tectonics, large-scale surface mass redistribution, mean sea level change, Earth orientation parameters, reference frame, and coseismic and postearthquake deformation. In this study, we explore the feasibility of studying tidal displacements directly from the daily coordinate time series and assess the accuracy of the extracted tidal signals.

The diurnal and semidiurnal tidal displacements are not perfectly averaged to zero for the span of the processing session and remain in the daily or weekly coordinate time series as alias signals with longer periods ranging from about 2 weeks to 1 year, as confirmed by the simulated series. ${ }^{(25)}$ The analysis of the regional vertical common-mode error series of the CGPS solutions also revealed a significant spectral peak at the period of 14 days (alias period of the M2 tide), which might be caused by the unmodeled M2 tidal displacements. ${ }^{(26,27)}$ Penna et al. and others proposed an alias harmonic analysis to estimate the alias signals from the daily CGPS coordinate time series through conventional harmonic analysis and then to convert them to the original tidal displacement signals. ${ }^{(25,28)}$ They demonstrated the feasibility of this approach to extract signals of eight primary diurnal and semidiurnal tidal constituents using simulated daily coordinate series. ${ }^{(13,25)}$ Later, Penna et al. extracted the alias tidal signatures from real CGPS daily height time series spanning seven years. ${ }^{(28)}$

In daily solutions, these diurnal and semidiurnal tidal signatures appear as multiple tiny slowly varying harmonics with their alias periods, which are determined by the beat period between the tidal period and the processing session or repeat orbit period. ${ }^{(25)}$ Besides the alias harmonic analysis, there is another flexible approach called frequency mixing, which transforms all periodic signals embedded in the time series into a series of different periodic signals with distinct frequencies while keeping the peak and phase characteristics of the original signals. It was originally used in such fields of microwave communications, radio astronomy, radar, plasma physics, remote control, remote sensing, and electronic countermeasures. ${ }^{(29-33)}$ For example, the approach used by Herring and Dong ${ }^{(3)}$ and Bohm et al. ${ }^{(34)}$ to estimate the highfrequency ERPs belonged to the frequency mixing method with the mean sidereal frequency as the distinct frequency. However, they applied frequency mixing during the processing session, which required data reprocessing and code modification. So far, to the authors' knowledge, this method has not been used to detect diurnal and semidiurnal tide displacements directly from the daily solutions for the end users.

In this study, we verify the feasibility of the frequency mixing method for extracting the diurnal and semidiurnal tidal displacements from the daily CGPS coordinate time series, and 
compare the method with the alias harmonic analysis. Since the OTL effects in the height direction are much larger than those in the east and west directions, ${ }^{(10)}$ for brevity, in the rest of this paper, we only focus on the height component of the GPS coordinate time series. In the following Sects. 2 and 3, details of the alias harmonic analysis and frequency mixing methods are given, respectively. The simulation data are analyzed through both of the approaches described in Sect. 4, and the real CGPS daily vertical residual time series from eight selected globally distributed stations are used to test whether the diurnal and semidiurnal tidal displacements can be extracted through the two above-mentioned methods described in Sect. 5. Finally, the discussion and conclusions are presented in Sect. 6.

\section{Alias Harmonic Analysis}

In signal processing, aliasing refers to the distortion or artifact, which results when the signal reconstructed from samples is different from the original continuous signal. For example, to correctly reconstruct a signal with a known frequency $f$, the sample frequency $f_{s}$ should be higher than the Nyquist frequency $f_{N}=2 f$. If not, aliasing occurs.

The alias frequency is calculated using ${ }^{(25)}$

$$
f^{\prime}=\operatorname{abs}\left[f-\frac{1}{\Delta} \operatorname{int}(f \Delta+0.5)\right]
$$

where $f^{\prime}$ means the alias frequency, $f$ represents the original frequency of the signal, $\Delta$ is the sampling interval, and the "int" function returns the largest integer less than or equal to its argument.

Penna and Stewart have demonstrated that the unmolded diurnal and semidiurnal tidal displacements will propagate to CGPS daily vertical time series as several spurious features with long periods. ${ }^{(25)}$ However, the estimated amplitudes, periods, and phases of these alias terms are easily affected by many systematic effects, such as those of the site position, orbital configuration, and processing session length, because they are very sensitive to such parameters as the period and amplitude of the input systematic error, the period and amplitude of the satellite orbit, the location of a GPS station relative to the satellite, and the length of the observation processing session window. In Table 1, we list the theoretical alias periods of diurnal and semidiurnal ocean tidal constituents, computed using Eq. (1), and the alias periods derived by Stewart and co-workers by the alias harmonic analysis of simulated CGPS data. ${ }^{(13,25)}$

Note that the periods of M2, S2, N2, O1, P1, and Q1 were defined in accordance with Melchior, ${ }^{(35)}$ and the periods of K1 and K2 were defined as WGS84 earth rotation periods. ${ }^{(36)}$ P-AP denotes the estimated alias periods derived by Penna and Stewart, resulting from the $24 \mathrm{~h}$ data processing and GPS constellation repeat orbit effects. ${ }^{(25)}$ S-AP denotes the estimated alias periods derived by Stewart et al., listed in the order of descending amplitude. ${ }^{(13)}$ 
Table 1

Alias periods of diurnal and semidiurnal tidal constituents.

\begin{tabular}{lccccc}
\hline \multirow{2}{*}{ Constituent } & Period (h) & \multicolumn{2}{c}{ P-AP (days) } & \multicolumn{2}{c}{ S-AP (days) } \\
\cline { 3 - 6 } & & 24 h Processing & Repeat orbit & $\begin{array}{c}\text { Maximum } \\
\text { amplitude }\end{array}$ & $\begin{array}{c}\text { Secondary } \\
\text { amplitude }\end{array}$ \\
\hline M2 & 12.42 & 14.76 & 13.66 & 13.66 & 14.76 \\
S2 & 12.00 & $\infty$ & 182.63 & 182.63 & 365.26 \\
N2 & 12.66 & 9.61 & 9.13 & & \\
K2 & 11.97 & 182.63 & $\infty$ & 13.66 & 14.19 \\
O1 & 25.82 & 14.19 & 13.66 & $\infty$ & 365.24 \\
K1 & 23.93 & 365.26 & $\infty$ & & \\
P1 & 24.07 & 365.24 & 182.63 & & \\
Q1 & 26.87 & 9.37 & 9.13 & &
\end{tabular}

\section{Frequency Mixing}

Frequency mixing means that signals are multiplied together to generate new frequencies. It enables signals to be converted from one frequency to another so that signal processing, for example, can be undertaken at a low or high frequency, whichever is easier to perform.

Assume there is an original high-frequency signal like Eq. (2), where $a$ is the amplitude, $\omega$ is the frequency, $t$ is the sampling interval, and $\varphi$ represents the initial phase.

$$
A(t)=a e^{i(\omega t+\varphi)}
$$

To convert the frequency of the original signal, we multiply it with a fixed frequency signal $\cos \omega_{0} t$. Then the original signal becomes a new combined signal that can be transformed into two equal-amplitude parts, the sum frequency component $\omega+\omega_{0}$ and the difference frequency component $\omega-\omega_{0}$, as shown by

$$
A^{\prime}(t)=\frac{1}{2} a e^{i\left[\left(\omega+\omega_{0}\right) t+\varphi\right]}+\frac{1}{2} a e^{i\left[\left(\omega-\omega_{0}\right) t+\varphi\right]} .
$$

Compared with the original signal, the sum frequency component of the new combined signal becomes a higher-frequency signal, whereas the difference frequency component is converted to a low-frequency signal. The amplitudes of both components are half the amplitude of the original signal. After using a band-pass filter to isolate the sum frequency component, the corresponding original high-frequency signal can be detected through the difference frequency component. However, this method has a basic problem, the image frequency.

Assuming the component at the frequency of $\omega-\omega_{0}$ is what we want to find in the spectrum, we presume that it corresponds to the original frequency $\omega$ after being mixed with the frequency $\omega_{0}$. However, as shown in Eq. (3), which includes the component of $\cos \left(\omega-\omega_{0}\right) t$, since cosine is an even function, the component at the frequency of $\omega-\omega_{0}$ may also come from 
another frequency $\omega_{i m g}=\omega-\omega_{0}$, which is called the image frequency. For example, to detect an original high-frequency signal at about $20 \mathrm{MHz}$, there are two fixed frequency signals that can generate a $1 \mathrm{MHz}$ difference frequency: 21 and $19 \mathrm{MHz}$. The latter fixed frequency of 19 $\mathrm{MHz}$ is called the image frequency.

There are two ways to avoid the appearance of the image frequency. The first one is to use a special method that does not respond to the image frequency. The second one is to choose an appropriate multiplied fixed frequency signal to minimize the possibility of image sensitivity. As the first approach is more complex and thus unpopular, in this work, we simply choose the second approach to reduce the effect of the image frequency.

\section{Simulation Data Analysis}

To verify the frequency mixing method mentioned above, we simulated a 12 year daily vertical time series, which contain the diurnal tidal components plus Gaussian noise, as shown in Table 2. The semidiurnal components can be handled in the same way.

The power spectrum obtained by Welch's modified periodogram method is shown in Fig. 1. The Hamming window is applied with the same length of segments, the overlap between the segments is $50 \%$, and the number of Fast Fourier Transform (FFT) points (nfft) is the next power of two greater than or equal to the length of the segment. To avoid misidentification at

Table 2

Constituents of the simulation data.

\begin{tabular}{lcccc}
\hline Constituent & $\begin{array}{c}\text { Period } \\
(\mathrm{h})\end{array}$ & $\begin{array}{c}\text { Phase } \\
(\mathrm{deg})\end{array}$ & $\begin{array}{c}\text { Amplitude } \\
(\mathrm{mm})\end{array}$ & $\begin{array}{c}\text { Noise } \\
(\mathrm{mm})\end{array}$ \\
\hline K1 & 23.93 & 0 & 8 & 1 \\
O1 & 25.82 & 0 & 5 & 1 \\
P1 & 24.07 & 0 & 3 & 1 \\
Q1 & 26.87 & 0 & 2 & 1 \\
\hline
\end{tabular}

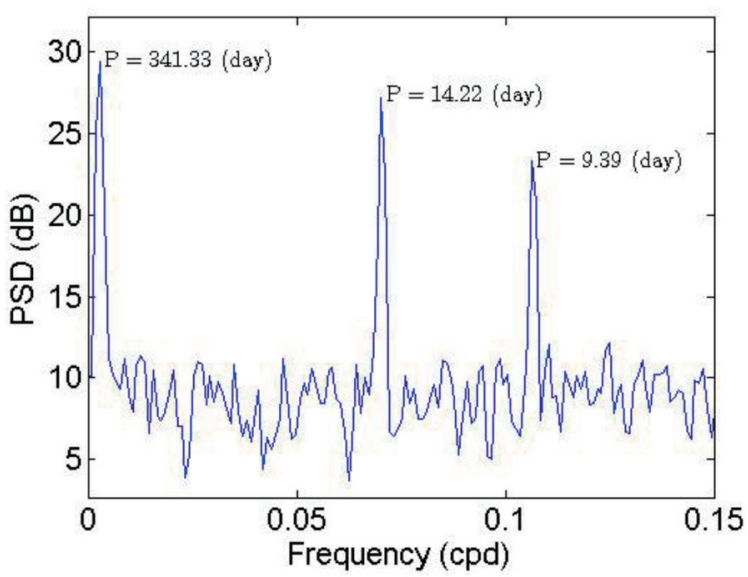

Fig. 1. (Color online) Power spectrum obtained by Welch's method for four segments and the simulated 12 year daily vertical time series with three peaks labeled. The 9.39, 14.22, and 341.33 day periods correspond to Q1, O1, and $\mathrm{K} 1$ or P1 respectively. The unit of frequency is cycles per day (cpd). 
the alias period of about 1 year, we adopted a segment number of 8 , so as to make the effective length of the segment more than 2.5 years, as suggested by Blewitt and Lavallée. ${ }^{(37)}$ One can find that the alias periods for $\mathrm{O} 1$ and Q1 as listed in Table 1 are clearly present in the spectra, while the alias periods of $\mathrm{K} 1$ and $\mathrm{P} 1$ are slightly shifted owing to the limited frequency resolution. This suggests that the method used to extract diurnal tidal signals from the daily time series using the alias harmonic analysis mentioned in Sect. 2 is not appropriate for K1 and $\mathrm{P} 1$, whose theoretical alias frequencies are about 1 year.

Figure 2 shows the power spectra of the data simulated by frequency mixing with different fixed frequency signals. The diurnal tidal signals are clearly detected, and their corresponding image frequencies are distributed on the other side of fixed frequency. With a fixed signal with a period of $22 \mathrm{~h}$, the wanted diurnal and semidiurnal tidal signals are well separated with the alias frequencies of the sum frequency signals, which are easy to remove using a low-pass filter. However, if we adopt a fixed signal with a period of $23 \mathrm{~h}$, the unwanted alias signals will be inside the frequency band of the wanted tidal signals, e.g., there is an alias frequency between the $\mathrm{O} 1$ and $\mathrm{P} 1$ frequencies. This suggests that an appropriate multiplied fixed frequency signal for minimizing the possibility of image sensitivity is important for frequency mixing.

\section{Real CGPS Vertical Residual Time Series Analysis}

\subsection{Data set and processing}

To find diurnal and semidiurnal tidal displacements in the real CGPS time series using the frequency mixing method, we selected eight globally distributed CGPS stations, as listed in Table 3. The geographical distribution of these stations is shown in Fig. 3. NICO, NKLG, SHAO,

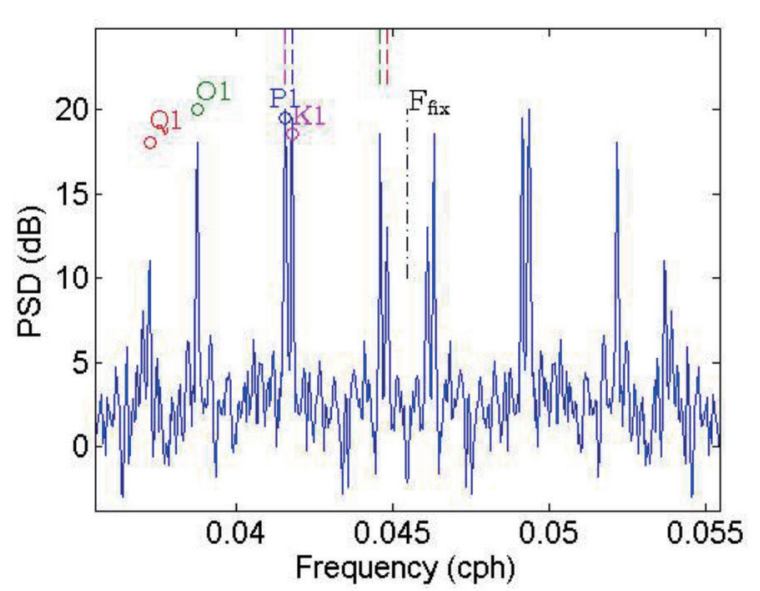

(a)

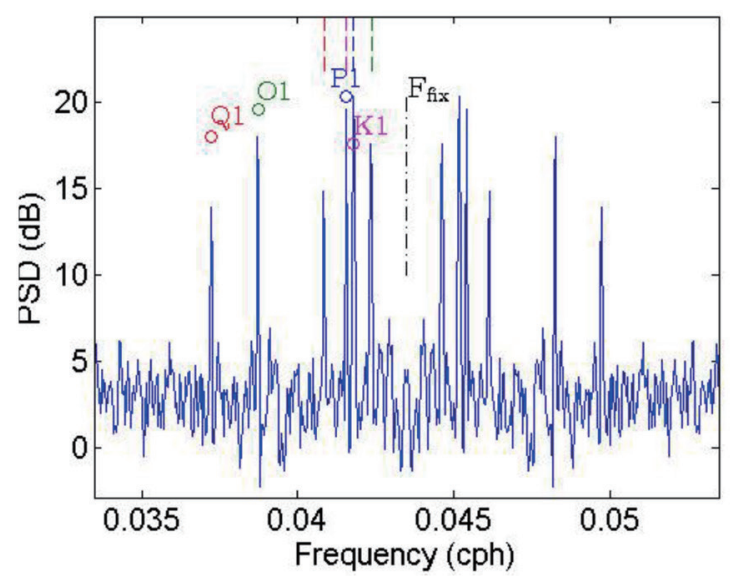

(b)

Fig. 2. (Color online) (a) Left panel: Power spectrum obtained by Welch's method for the simulated 12 year daily height time series using the frequency mixing method with a fixed frequency signal at the period of $22 \mathrm{~h}$, labeled with a dash-dotted line. The clearly detected tidal constituents are labeled with colorful characters. The colored dashed lines denote the alias frequency of the up-converted image frequency of the tidal constituents labeled with the same color. (b) The same as the left panel but with a fixed signal at the period of $23 \mathrm{~h}$. The unit of frequency is cycles per hour (cph). 
Table 3

Time series of eight CGPS stations.

\begin{tabular}{lrccc}
\hline Site & Lat $\left(^{\circ}\right)$ & Long $\left(^{\circ}\right)$ & Time range $($ year) & Data availability (\%) \\
\hline NICO & 33.40 & 35.14 & $2008.00-2018.00$ & 96.8 \\
NKLG & 9.67 & 0.35 & $2011.40-2018.00$ & 90.3 \\
SHAO & 121.20 & 31.10 & $2008.00-2014.36$ & 98.1 \\
CHUR & 265.91 & 58.76 & $1995.13-2018.00$ & 81.8 \\
BRST & 355.50 & 48.38 & $2011.00-2018.00$ & 95.8 \\
KARR & 117.10 & -20.98 & $2008.00-2014.80$ & 96.7 \\
KOKB & 200.33 & 22.12 & $2008.00-2015.00$ & 97.4 \\
DGAR & 72.37 & -7.27 & $2008.23-2015.00$ & 95.8 \\
\hline
\end{tabular}

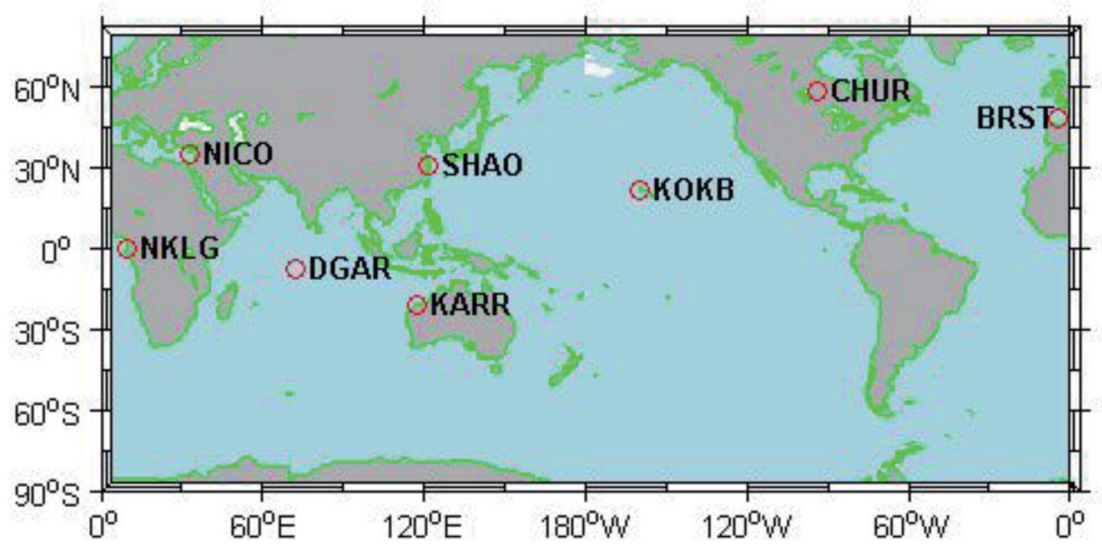

Fig. 3. (Color online) Geographic locations of eight IGS stations

CHUR, BRST, and KARR are located in continental coastal regions, while KOKB and DGAR are on deep ocean islands.

The daily coordinate time series of these stations are provided by Scripps Orbit and Permanent Array Center (SOPAC) (http://garner.ucsd.edu), using a combination of independent analyses performed by Jet Propulsion Laboratory (JPL) and Scripps Institution of Oceanography (SIO) (http:/geoapp03. ucsd.edu/gridsphere/gridsphere). The time series were generated using an elevation cutoff angle of $10^{\circ}$ and a $30 \mathrm{~s}$ sampling interval during the $24 \mathrm{~h}$ processing sessions (with fix ambiguities). The new Global Mapping Function (GMF) was selected to estimate the tropospheric zenith path delay. All the long-term ocean tidal components (Mf, Mm, and Ssa), the principal diurnal and semidiurnal tidal signatures (M2, S2, N2, K2, K1, O1, P1, and Q1) and the solid Earth tide effects, are removed using the FES2004 ocean tide and standard models advocated in the IERS Conventions 2010, respectively. The atmosphere loading effects are not removed.

There are no large gaps in the time series of all stations, and the time series lengths of most stations are about 6-10 years, except for CHUR, which has the longest time series of about 23 years. The constant offsets, linear trends, coseismic jumps, nonseismic jumps, postseismic deformation, and local polynomial terms are removed from these time series using the 
Quasi-observation Combination Analysis (QOCA) software. ${ }^{(26,38)}$ The seasonal (annual and semiannual) terms are reserved, since part of the annual and semiannual terms might account for the high-frequency alias signals. Although the data availability of the selected stations shown in Table 3 is generally greater than $90 \%$, the small time gaps in the time series will still affect its harmonic analysis. In order to minimize the effect, firstly, we use the linear interpolation method to interpolate the daily vertical residual time series to obtain a new evenly spaced time series. The new time series are then analyzed by alias harmonic analysis (at the theoretic alias periods in Table 1) and frequency mixing (at the original theoretic periods in Table 1) to estimate the unmolded eight principal diurnal and semidiurnal tidal displacements, respectively. The results are displayed in Figs. 4 and 5.

\subsection{Interpretation of the results}

As shown in Fig. 4, the spectra of the data from the eight stations present prominent separate spectral peaks near the alias frequencies of $\mathrm{K} 1$ and $\mathrm{P} 1$. The other tidal signatures are negligible, except for K2. The second primary spectral peaks of the CHUR station, with the longest time series of 23 years, demonstrate the existence of the semidiurnal tidal signal K2. Since the theoretical alias period of S2 is infinite, it is theoretically impossible to extract this tidal signal by alias harmonic analysis, unless there is a slight frequency shift, which would change its real alias frequency to about half a year as shown in Table 1, owing to other factors, such as the site position, orbital configuration, and processing session length. ${ }^{(13)}$

Figure 5 shows the power spectrum for each station obtained by frequency mixing. The diurnal tidal displacement constituents $\mathrm{P} 1$ and $\mathrm{K} 1$ and the semidiurnal signals S2 and K2 are clearly detected and well separated. The detected P1 and K1 are consistent with their theoretic tidal frequencies, whereas there is a frequency shift for S2 and K2. In addition, S2 cannot be detected by alias harmonic analysis, and $\mathrm{K} 2$ is marginally detected in the longest time series. This suggests that frequency mixing is more effective than alias harmonic analysis for extracting the diurnal and semidiurnal tidal displacements from daily vertical residual time series.

The power spectra obtained by frequency mixing indicates the existence of the diurnal and semidiurnal tidal displacements at all eight stations, although this was modeled by the FES2004 ocean tide model. Table 4 lists the modeled amplitudes of the diurnal and semidiurnal tidal constituents that have been removed from the original time series, and the estimated residual vertical amplitude. The modeled vertical amplitudes of the tidal effects at each station are calculated using the SPOTL software (https://igppweb.ucsd.edu/ agnew/Spotl/spotlmain.html), in the instantaneous center of mass (CM) reference frame, and convolved with the ocean tide model FES2004 with Green's function. ${ }^{(39)}$ The residual vertical amplitudes of K1, P1, S2, and $\mathrm{K} 2$, detected by the frequency mixing method, are estimated at their difference frequency by least squares fitting. ${ }^{(19)}$ The amplitude squeezing effect has been considered since the CGPS daily time series is an average solution of the station coordinates. The amplitudes of diurnal and semidiurnal tidal displacements after considering the amplitude squeezing effect $A_{j}{ }^{\prime}$ are calculated using Eq. (4), ${ }^{(40)}$ where $f_{j}$ is the frequency of the periodic signal $j, A_{j}$ represents the 

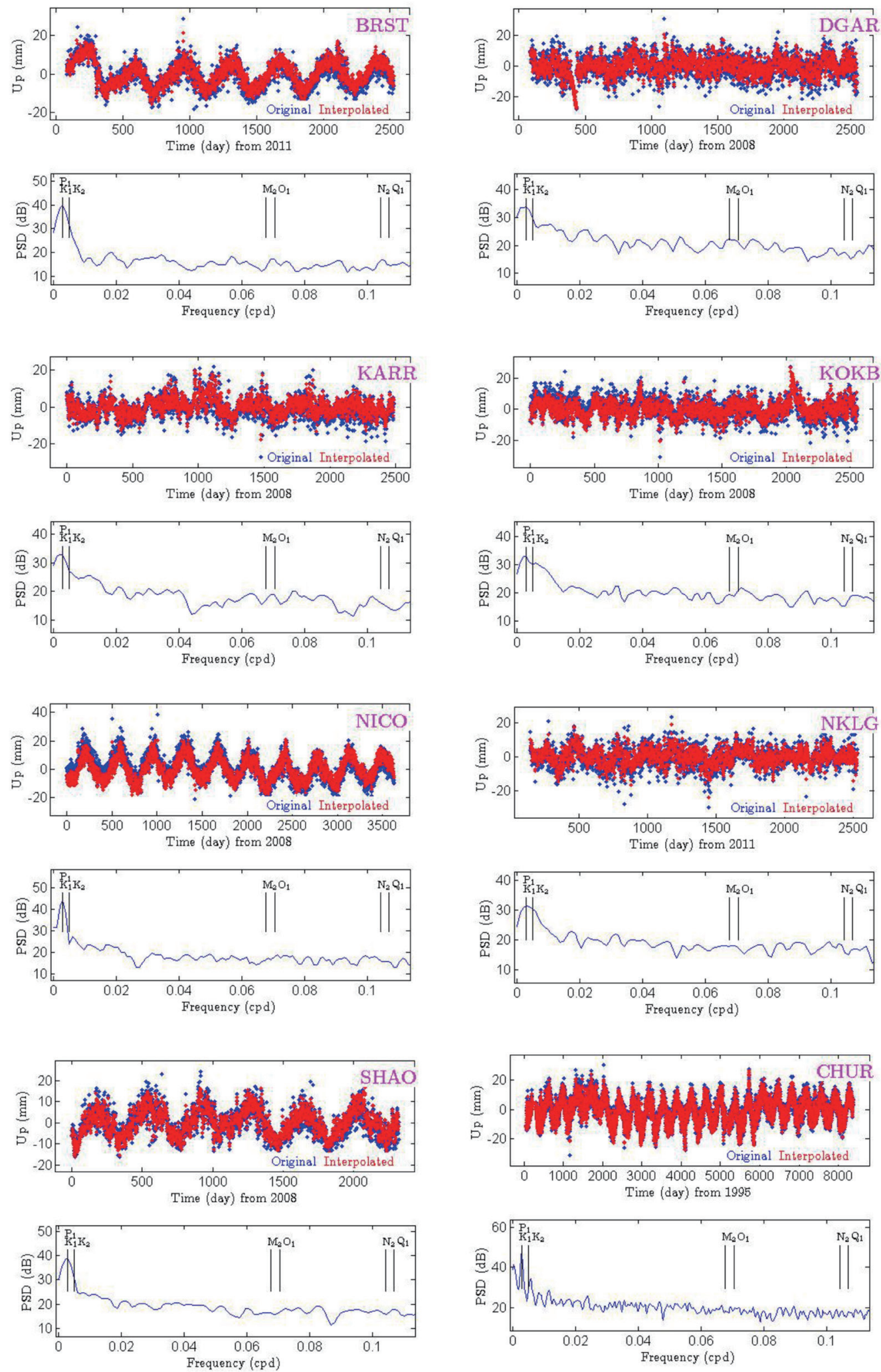

Fig. 4. (Color) CGPS daily vertical residual time series and power spectra for data from eight IGS stations. For each station, the upper panel shows the original (blue) and interpolated (red) time series, and the site name is labeled in the right top corner; the lower panel shows the power spectrum with the theoretical alias frequencies labeled. The alias frequencies of $\mathrm{K} 1$ and $\mathrm{P} 1$ are too close to be resolved in the plot. 

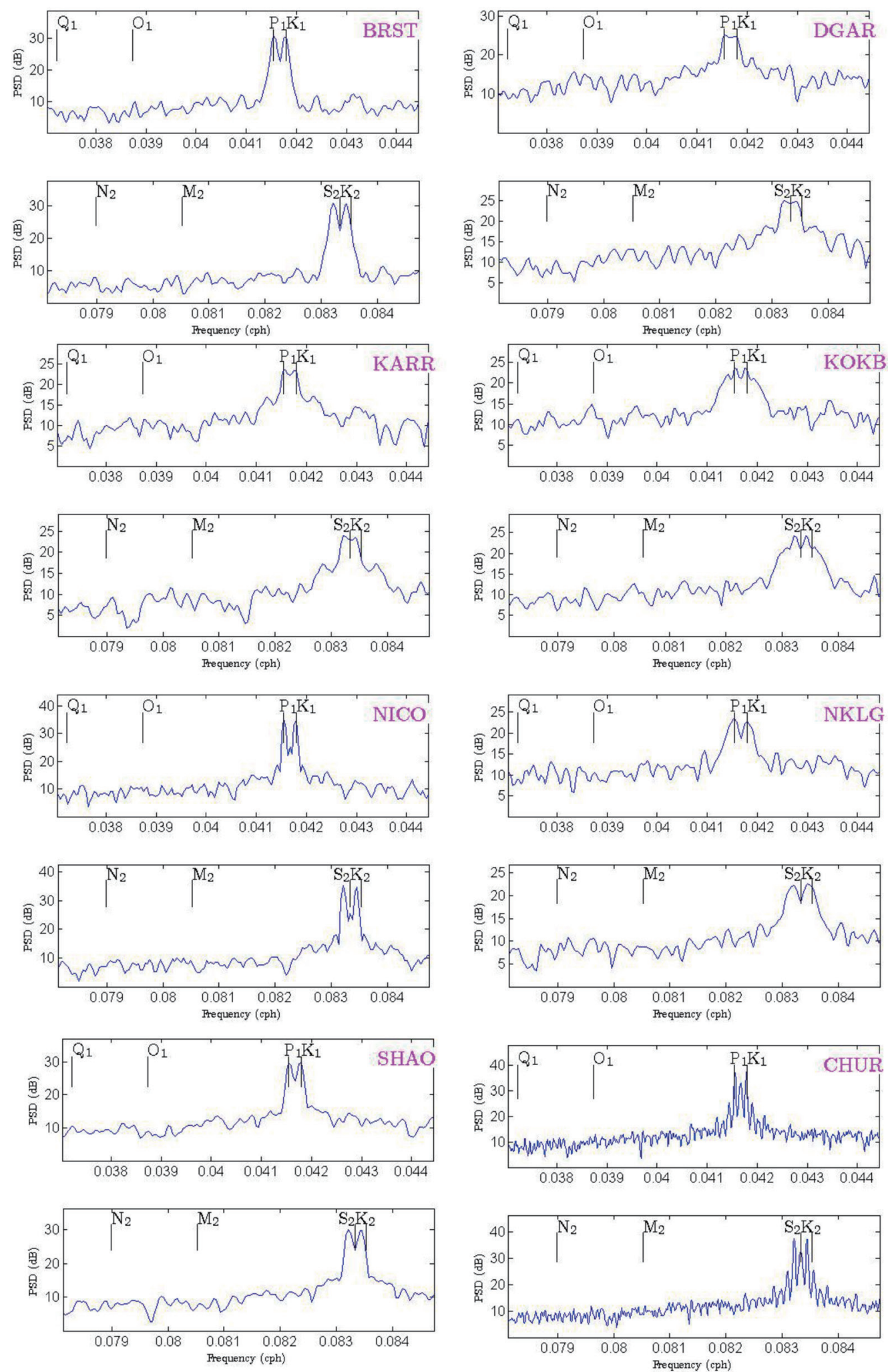

Fig. 5. (Color online) Power spectra for data from eight IGS stations obtained by frequency mixing. For each station, the upper and lower panels show frequency bands of the diurnal and semidiurnal signals, respectively. The frequency of each tidal constituent is labeled. 
Table 4

Ocean-tide-modeled and CGPS-estimated tidal displacement vertical amplitudes.

\begin{tabular}{|c|c|c|c|c|c|c|c|c|c|c|c|c|}
\hline \multirow{2}{*}{ Site } & \multicolumn{3}{|c|}{$\mathrm{S} 2(\mathrm{~mm})$} & \multicolumn{3}{|c|}{$\mathrm{K} 2(\mathrm{~mm})$} & \multicolumn{3}{|c|}{$\mathrm{K} 1 \mathrm{(mm})$} & \multicolumn{3}{|c|}{ P1 (mm) } \\
\hline & M-AM & D-AM & PER & M-AM & D-AM & PER & M-AM & D-AM & PER & M-AM & D-AM & PER \\
\hline $\mathrm{NICO}$ & 1.13 & 0.21 & $15.8 \%$ & 0.31 & 0.21 & $40.5 \%$ & 0.59 & 3.85 & $86.7 \%$ & 0.20 & 4.46 & $95.7 \%$ \\
\hline NKLG & 6.83 & 0.21 & $3.0 \%$ & 1.75 & 0.21 & $10.7 \%$ & 3.32 & 0.61 & $15.5 \%$ & 1.09 & 0.81 & $42.7 \%$ \\
\hline SHAO & 1.51 & 0.00 & $0.0 \%$ & 0.23 & 0.84 & $78.6 \%$ & 6.75 & 4.05 & $37.5 \%$ & 2.18 & 4.46 & $67.2 \%$ \\
\hline CHUR & 2.29 & 1.27 & $35.6 \%$ & 0.36 & 0.63 & $63.7 \%$ & 2.84 & 1.01 & $26.3 \%$ & 0.98 & 0.81 & $45.3 \%$ \\
\hline BRST & 13.68 & 0.00 & $0.0 \%$ & 3.27 & 0.84 & $20.5 \%$ & 4.40 & 4.25 & $49.2 \%$ & 1.45 & 4.87 & $77.1 \%$ \\
\hline KARR & 8.26 & 0.00 & $0.0 \%$ & 1.87 & 0.21 & $10.1 \%$ & 10.02 & 2.03 & $16.8 \%$ & 3.31 & 2.23 & $40.3 \%$ \\
\hline KOKB & 8.26 & 0.42 & $4.9 \%$ & 1.87 & 0.63 & $25.3 \%$ & 10.02 & 1.42 & $12.4 \%$ & 3.31 & 1.22 & $26.9 \%$ \\
\hline DGAR & 16.36 & 0.42 & $2.5 \%$ & 4.14 & 0.21 & $4.8 \%$ & 2.13 & 2.43 & $53.3 \%$ & 0.70 & 2.64 & $79.0 \%$ \\
\hline
\end{tabular}

Notes: M-AM means the modeled height amplitudes of the FES2004 ocean tide model, D-AM means the estimated residual amplitudes from the least square fit to the time series, and PER means the percentage of the estimated residual amplitudes relative to the real amplitude under the hypothesis that the real amplitude is the sum of the modeled and estimated vertical amplitudes.

estimated amplitude from the daily vertical residual time series, and $\Delta t$ represents the data processing session.

$$
A_{j}^{\prime}=A_{j} \frac{\sin \left(\pi f_{j} \Delta t\right)}{\pi f_{j} \Delta t}
$$

As listed in Table 4, for most stations, $\mathrm{K} 1$ and $\mathrm{P} 1$ are the larger tidal displacements in the estimated four tidal displacements, with amplitudes of $0.61-4.87 \mathrm{~mm}$, and $\mathrm{S} 2$ is the smallest. Assuming the sum of the modeled and estimated vertical amplitudes is the real amplitude, the percentage of the estimated residual amplitude denotes the relative uncertainty of the tide model. The ratio of the estimated residual S2 amplitude is less than $35.6 \%$ for each station, while the percentages for $\mathrm{P} 1, \mathrm{~K} 2$, and $\mathrm{K} 1$ can reach 95.7, 86.7, and 78.6, respectively. This implies an inconsistency between CGPS observed results and the FES2004 ocean tide model outputs, especially for the components of P1, K1, and K2.

\section{Discussion and Conclusion}

We analyze eight globally distributed CGPS daily vertical residual time series by alias harmonic analysis and frequency mixing. The time series lengths of most stations are about 6-10 years, except for CHUR, which has the longest time series of about 23 years. The prominent separate spectral peaks displayed in Figs. 4 and 5 indicate the feasibility of these two methods and the existence of these unmolded tidal displacements.

The detected four prominent tidal displacements illustrate the superiority of the frequency mixing method, which is capable of detecting and efficiently separating the diurnal and semidiurnal tidal signatures S2, K1, P1, and K2. Although the alias harmonic analysis can detect $\mathrm{K} 1$ and P1, separating them is impossible. K2 and S2 cannot be detected by alias harmonic analysis, unless other factors affecting them are considered and there is more than twenty years of long daily coordinate time series existing, such as the case of the CHUR station. 
Recent advances in space geodesy have demonstrated that the lunar-only tidal constituents M2, N2, O1, and Q1 obtained from the real CGPS observation of tidal displacements coincide well with ocean tide model estimates. The discrepancies between the CGPS and model estimates for the solar-related constituents $\mathrm{S} 2, \mathrm{~K} 1, \mathrm{P} 1$, and K2 are relatively large. ${ }^{(20,41)}$ The M2, $\mathrm{N} 2$, O1, and Q1 tidal displacements not detected by frequency mixing also demonstrate that the FES2004 ocean tide model is more favorable for the lunar-only tidal constituents.

A previous study revealed that the vertical uncertainty of different tide models can be up to $8 \mathrm{~mm}$ in some coastal regions. ${ }^{(9)}$ This is quite consistent with our amplitude of $4.87 \mathrm{~mm}$ estimated by frequency mixing using CGPS daily residual vertical time series. The detected residual amplitude percentages of $\mathrm{K} 2, \mathrm{P} 1$, and $\mathrm{K} 1$, as listed in Table 4, compared with S2, are relatively large, which is also consistent with the conclusion of Yuan and co-workers, ${ }^{(9,20)}$ who documented that, in vertical components, the RMS between the CGPS observed tidal displacements and model estimations for K2, P1, and K1 are relative larger than that for S2.

At present, the mechanisms responsible for the discrepancies in these constituents between the CGPS observations and model estimates are still inconclusive. King ${ }^{(42)}$ and Zhong et al. ${ }^{(43)}$ suggested in their previous reports, that the majority of the CGPS observation-versus-model tidal displacement residuals of K1 and K2 may be due to the CGPS satellite orbit errors and multipath effects. Furthermore, King and Williams proposed that satellite orbit mismodeling and CGPS signal propagation effects (including tropospheric and higher-order ionospheric effects) are more serious than local site effects such as multipath. ${ }^{(44)}$

The detected tidal displacements of $\mathrm{K} 1$ and $\mathrm{K} 2$ in Fig. 5 appear exactly at their theoretic frequencies, while there appears to be a frequency shift for S2 and K2. In agreement with a similar simulation described in Sect. 4 with the detected 4 tidal constituents, we found that the peaks close to the frequencies of S2 and K2 actually originate from the alias signal due to the image of the alias frequency of P1 and K1 after frequency mixing, which can be calculated using

$$
f_{\text {img }}^{\prime}=-\operatorname{abs}\left[\left(f-f_{f i x}\right)-\frac{1}{\Delta} \operatorname{int}\left(\left(f-f_{f i x}\right) \Delta+0.5\right)\right]+f_{f i x},
$$

where $f_{\text {fix }}$ is the fixed frequency mixed with the original frequency $f$ and $f_{\text {img }}^{\prime}$ is the image alias frequency of $f-f_{f i x}$ added to $f_{f i x}$. This suggests that both the diurnal and semidiurnal tidal constituents found in Fig. 5 are reliable.

Both the alias harmonic analysis and frequency mixing methods can directly use the existing daily coordinate time series, and are more convenient than the static and kinematic approaches, which require data reprocessing. However, the alias harmonic analysis method has some deficiencies. First, the estimated amplitudes, periods, and phases of these alias terms are easily affected by many systematic effects, such as the site position, orbital configuration, and processing session length. ${ }^{(13)}$ Second, the detected annual and semiannual alias signatures may be affected by other geophysical factors, such as the mismodeled solid Earth tide, atmospheric mass loading, snow and soil moisture mass loading, and under groundwater loading. ${ }^{(38)}$ 
We concluded that the frequency mixing method can be used for detecting the diurnal and semidiurnal tidal displacements from CGPS daily vertical time series. It is a technique effective for evaluating ocean tide effects using CGPS high precision daily solutions, which is sufficient to improve the GNSS data processing accuracy and provide high-precision three-dimensional spatial geographic information for the development of the smart city. The primary goal of this work was to verify the feasibility of the method. The improvement and application of this method to regional or globally distributed sites shoud be further investigated.

\section{Acknowledgments}

We are grateful to Scripps Orbit and Permanent Array Center (SOPAC), Jet Propulsion Laboratory (JPL), and Scripps Institution of Oceanography (SIO) for providing daily coordinate time series of CGPS stations. This work is sponsored by the National Key R\&D Program of China (No. 2017YFE0100700), the National Natural Science Foundation of China (No. 41771475), and the Fund of Director of Key Laboratory of Geographic Information Science (Ministry of Education), East China Normal University (Grant No. KLGIS2017C01).

\section{References}

1 W. M. Kaula: Rev. Geophys. Space Phys. 2 (1964) 661. https://doi.org/10.1029/RG002i004p00661

2 H. Schuh and L. Moehlmann: Geophys. Res. Lett. 16 (1989) 1105. https://doi.org/10.1029/GL016i010p01105

3 T. A. Herring and D. Dong: J. Geophys. Res. 99 (1994) 18051. https://doi.org/10.1029/94JB00341

4 M. M. Watkins and R. J. Eanes: Adv. Space Res. 13 (1993) 251. https://doi.org/10.1016/0273-1177(93)90227-3

5 K. M. Larson, P. Cervelli, M. Lisowski, A. Miklius, P. Segall, and S. Owen: J. Geophys. Res. 106 (2001) 19. https://doi.org/10.1029/2001JB000305

6 S. A. Khan and H. G. Scherneck: J. Geod. 77 (2003) 117. https://doi.org/10.1007/s00190-003-0312-y

7 M. S. Bos and T. F. Baker: J. Geod. 79 (2005) 50. https://doi.org/10.1007/s00190-005-0442-5

8 G. Petit and B. Luzum: IERS Conventions IERS Technical Note 36 (2010) 11. https://www.iers.org/ SharedDocs/Publikationen/EN/IERS/Publications/tn/TechnNote36/tn36.pdf

9 L. G. Yuan, X. L. Ding, P. Zhong, W. Chen, and D. F. Huang: J. Geod. 83 (2009) 999. https://doi.org/10.1007/ s00190-009-0319-0

10 N. T. Penna, M. S. Bos, T. F. Baker, and H. G. Scherneck: J. Geod. 82 (2008) 893. https://doi.org/10.1007/ s00190-008-0220-2

11 N. T. Penna, P. J. Clarke, M. S. Bos, and T. F. Baker: J. Geod. 120 (2015) 6523. https://doi. org/10.1002/2015JB011882

12 M. S. Bos, N. T. Penna, and T. F. Baker: J. Geophys. Res. 120 (2015) 6540. https://doi.org/ 10.1002/2015JB011884

13 M. P. Stewart, N. T. Penna, and D. D. Lichti: J. Geod. 79 (2005) 479. https://doi.org/10.1007/s00190-005-04786

14 T. Ito, M. Okubo, and T. Sagiya: J. Geod. 48 (2009) 253. https://doi.org/10.1016/j.jog.2009.09.012

15 H. Schuh: IEEE Trans. Instrum. Meas. 38 (1989) 676. https://doi.org/ 10.1109/19.192375

16 O. J.Sovers: Geophys. Res. Lett. 21 (1994) 357. https://doi.org/10.1029/93GL02648

17 M. S. Schenewerk, J. Marshall, and W. Dillinger: J. Geod. Soc. Jpn. 47 (2001) 237. https://doi.org/10.11366/ sokuchi1954.47.237

18 C. R. Allinson, P. J. Clarke, S. J. Edwards, M. A. King, T. F. Baker, and P. R. Cruddace: Geophys. Res. Lett. 31 (2004) 5603. https://doi.org/10.1029/2004GL020588

19 M. A. King, N. T. Penna, P. J. Clarke, and E. C. King: J. Geophys. Res. 110 (2005) B08401. https://doi. org/10.1029/2004JB003390

20 L. Yuan, B. F. Chao, X. Ding, and P. Zhong: J. Geophys. Res. 118 (2013) 2618. https://doi.org/10.1002/ jgrb.50159

21 S. A. Khan and C. C. Tscherning: Geophys. Res. Lett. 28 (2001) 2249. https://doi.org/10.1029/2000GL011890 
22 M. Vergnolle, M. N. Bouin, L. Morel, F. Masson, S. Durand, J. Nicolas, and S. A. Melachroinos: Geophys. J. Int. 173 (2008) 444. https://doi.org/10.1111/j.1365-246X.2008.03734.x

23 S. A. Melachroinos, R. Biancle, M. LIubes, E. Perosanz, F. Lyard, M. Vergnolle, M. N. Bouin, F. Masson, J. Nicolas, L. Morel, and S. Durand: J. Geod. 82 (2008) 357. https://doi.org/10.1007/s00190-007-0185-6

24 T. Ito and M. Simons: Science 332 (2011) 947. https://doi.org/10.1126/science.1202584

25 N. T. Penna and M. P. Stewart: Geophys. Research Lett. 30 (2003) 2184. https://doi.org/10.1029/2003GL018828

26 D. Dong, P. Fang, Y. Bock, F. Webb, L. Prawirodirdjo, S. Kedar, and P. Jamason: J. Geophys. Res. B: Solid Earth. 111 (2006) 3405. https://doi.org/10.1029/2005JB003806

27 H. Dragert, T.S. James, and A. Lambert: Geophysical Res. Letter. 27 (2000) 2045. https://doi.org/ doi:10.1029/2000GL011536

28 N. T. Penna, M. A. King, and M. P. Stewart: J. Geophys. Res. 112 (2007) B02402. https://doi. org $/ 10.1029 / 2005 J B 004047$

29 D. C. Agnew: J. Geophys. Res. 102 (1997) 5109. https://doi.org/10.1029/96JB03458

30 S. K. Sunter: US Patent No. 20030071606 (2003). http://www.freepatentsonline.com/y2003/0071606

31 D. G. Lancaster and J. M. Dawes: Opt. Commun. 120 (1995) 307. https://doi.org/10.1016/00304018(95)00429-C

32 J. Chen and S. Quegan: IEEE Trans. Geosci. Remote Sen. 49 (2011) 2712. https://doi.org/10.1109/ TGRS.2011.2109065

33 S. Taravati: Phys. Rev. B 97 (2018) 115131. https://doi.org/10.1103/PhysRevB.97.115131

34 S. Böhm, A. Brzeziński, and H. Schuh: J. Geodyn. 62 (2012) 56. https://doi.org/10.1016/j.jog.2011.10.002

35 P. J. Melchior: The Earth Tides (1966).

36 NIMA (National Imagery and Mapping Agency), Dept. Defense World Geodetic System 1984, its definition and relationships with local geodetic systems, Technical Report TR8350.2, 3rd ed., 1997.

37 G. Blewitt and D. Lavallée: J. Geophys. Res. 107 (2002) 2145. https://doi.org/10.1029/2001JB000570.

38 D. Dong, P. Fang, Y. Bock, M.K. Cheng, and S. Miyazaki: J. Geophys. Res. 107 (2002) 2075. https://doi. org/10.1029/2001JB000573

39 G. A. Jacobs, G. H. Born, M. E. Parke, and P. C. Allen: J. Geophys. Res. 97 (1992) 17. https://doi. org/10.1029/92JC01708

40 D. W. Zheng: Chin. Astron. 3 (1979) 114. https://doi.org/10.1016/0146-6364(79)90083-5

41 H. Zhao and Q. Zhang: Surv. Rev. 2 (2017) 1. https://0.1080/00396265.2017.1407392

42 M. King: J. Geodyn. 41 (2006) 77. https://doi.org/10.1016/j.jog.2005.08.019

43 P. Zhong, X. L. Ding, L. G. Yuan, Y. L. Xu, K. Kwok, and Y. Q. Chen: J. Geod. 84 (2010) 145. https://doi. org/10.1007/s00190-009-0352-Z

44 M. A. King and S. D. P. Williams: J. Geophy. Res. 114 (2009) B10403. https://doi.org/10.1029/2009JB006319

\section{About the Authors}

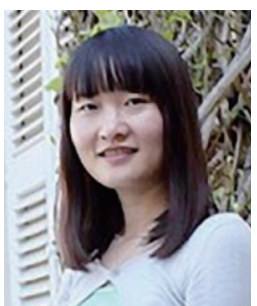

Yu Peng has been pursuing her Ph.D. degree in geophysics at East China Normal University since 2015. Her major research interests focus on the applications of high-precision GNSS in geophysics such as the land subsidence monitoring and mechanism analyses. (52151217004@stu.ecnu.edu.cn)

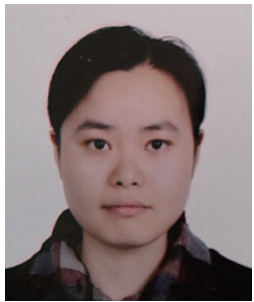

Wen Chen received her Ph.D. degree in geospatial information science (GIS) from East China Normal University in 2010. She currently works as an associate professor at the School of Information Science Technology, East China Normal University. Her major research interests include GNSS highprecision positioning and applications, spatial analysis, and GIS.

(wchen@sist.ecnu.edu.cn) 


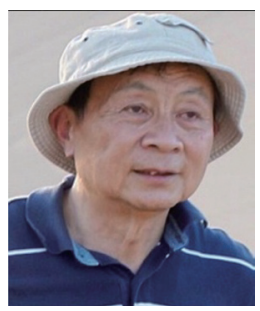

Danan Dong is currently a professor at East China Normal University. He obtained his Ph.D. degree in geophysics from Massachusetts Institute of Technology (MIT) and worked at the Jet Propulsion Laboratory (JPL) for 18 years. His main research interest is in the study of GNSS positioning and geophysics applying GPS technology. (dndong@cs.ecnu.edu.cn)

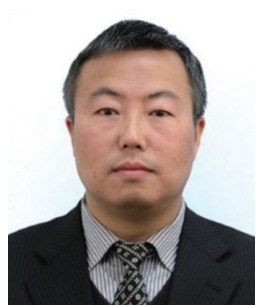

Chao Yu received his Ph.D. degree in physical electronics from Shanghai Institute of Technical Physics of Chinese Academy of Sciences in 2005. He currently works as a senior engineer at the School of Information Science Technology, East China Normal University. His major research interests include GNSS information processing and application.

(cyu@sist.ecnu.edu.cn)

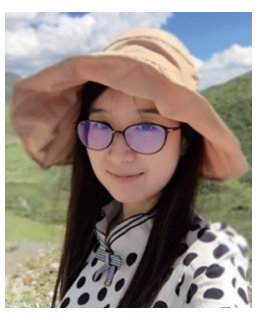

Zhiren Wang is currently a Ph.D. candidate at East China Normal University, Shanghai, China. Her interests include multipath mitigation and GNSS data processing. She has recently focused on developing algorithms for the detection and extraction of GPS non-line-of-sight signals.

(wangzhiren@163.com)

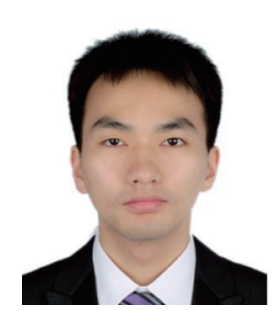

Jun Yan has been pursuing his Ph.D. degree in geophysics at East China Normal University since 2015. He is currently a visiting Ph.D. student at the University of California Berkeley, U.S.A. His major research interests include independent component analysis applications of GNSS time series in geophysics and detecting transient deformation in China and the United States. (yanjun1988@yahoo.com)

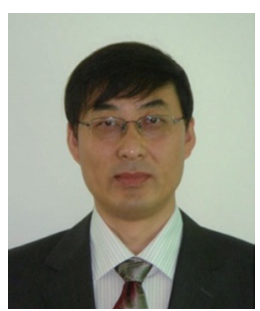

Min Liu received his Ph.D. degree in sedimentology from the Institute of Geology, Chinese Academy of Sciences in 1995. He currently works as a professor at the School of Geographic Sciences, East China Normal University. His major research interests include urban physical geography, natural hazards, and estuary biogeochemical cycles. (mliu@geo.ecnu.edu.cn) 